\title{
Development and characterization of gas sensors using thin films of polyaniline as active layer
}

\author{
André Luís Winck ${ }^{1}$, João Carlos Vernetti dos Santos ${ }^{1}$ \\ Denise Maria Lenz ${ }^{1}$, Douglas Milan Tedesco ${ }^{1}$
}

\author{
${ }^{1}$ Postgraduate Program in Materials Engineering and Sustainable Processes (PPGEMPS), Universidade Luterana do \\ Brasil (ULBRA), CEP: 92425-900, Canoas, Rio Grande do Sul, Brazil. \\ e-mail: jcvernetti@gmail.com, andreluiswinck@gmail.com, denise.lenz@gmail.com,douglas.m.tedesco@gmail.com
}

\begin{abstract}
This work aimed at the development and characterization of gas sensing devices using thin films of polyaniline (PAni) as active layer, deposited by in situ polymerization. The polyaniline, an intrinsically conductive polymer, was used due to its high sensitivity to gases, low response time and stability, ease of synthesis and low cost. The sensor was built on a glass fiber reinforced substrate with interdigitated gold electrodes coated with a PAni film layer whose thickness variation was fixed as a function of deposition time. The PAni characterization by Infrared Spectroscopy (FTIR) showed that the exposure to ethanol does not change the chemical structure of PAni. The thickness $(3.5 \mu \mathrm{m})$ and morphology of PAni films through Scanning Electron Microscopy (SEM) were compatible to those found in the literature. The electrical resistance of the sensors was evaluated as function of ethanol concentration and varied between 60 kohms for lower ethanol concentrations and $140 \mathrm{kohms}$ for higher concentrations. Nevertheless, a statistical analysis of the measurements in five sensors with different ethanol concentrations demonstrated that the sensors have not been able to differentiate ethanol concentrations to the level of significance of $5 \%$.
\end{abstract}

Keywords: gas sensor, ethanol, polyaniline, electrical conductivity

\section{INTRODUCTION}

Intrinsically conductive polymers (ICPs) have several properties with potential technological applications, including electromagnetic shielding [1], electro-mechanical sensors [2], chemical sensors [3,4] and biosensors [5]. These ICPs are characterized mainly by the ease of controlling the electrical conductivity, environmental stability and relatively simple synthesis. Nevertheless, these materials still have difficulty in processing, poor mechanical strength (compared to conventional polymers) and exhibit lower electrical conductivities values with respect to metals and materials such as carbon nanotubes and graphene [6].

One of the measures studied to improve the mechanical properties of ICP has been their mixture with conventional polymers, associating the processability of the matrix with the electrical properties of the ICP [7]. To increase the electrical conductivity of ICP, conductive nanofillers, such as carbon nanotubes (NTC) and graphene can be incorporated to an ICP composite matrix, resulting in electrical conductivity values in the range assigned to metals [8]. These processed materials find application in conductive inks, coatings, among others [6].

Polyaniline (PAni) is one of the most promising ICP for presenting thermal stability, processability and highest electrical conductivity among all ICP. It also presents economic advantage for mass production because of the simplicity of their synthesis [9]. These polymers are gaining attention from the scientific community since their electrical conductivity can be markedly altered in contact with a gas and this change can be accurately detected. Due to this feature, sensors based on PAni have been constructed and used on devices capable of producing different responses when exposed to different types of odorous substances as chemical sensors which were conventionally called electronic nose or e-nose [10,11]. However, the development of PAni sensors that preserve their quality while robustly enriching their gas-sensing features is still rare and remains challenging for the realization of rapid and sensitive gas detection [12]. Thus, further studies must be performed towards the reproducibility and stability of these sensors.

In this study, a gas sensor was developed to directly determine the concentration of ethanol (hydrous 
ethanol fuel) without the use of the instrumentation normally used like density gauges, thermometers and correction tables. The measurements of five different gas sensors constructed from chemically synthesized PAni in different conditions were discussed with respect to their electrical sensitivity to ethanol. In this way, this article contributes to the better understanding of factors and metrological parameters that affect the properties and response of PAni sensors to different stimulus sources [13].

\section{MATERIALS AND METHODS}

All the chemicals (aniline, ethanol, ammonium persulphate, hydrochloric acid) used were reagent grade and aniline was distilled under vacuum prior to use.

\subsection{Production of ethanol sensors}

An electronic printed circuit board (PCB) sensor substrate made of glass fiber reinforced epoxy resin with 1 mm thickness (FR4) was idealized with an array of interdigitated copper electrodes (It was built by the Casa do Circuito Company). Glass blades with interdigitated electrodes of ITO as substrate sensors are often used [14]. Figure 1 shows the layout of the designed PCB. The electrodes have a width of $0.17 \mathrm{~mm}$ and the spacings between them are $0.17 \mathrm{~mm}$. This spacing was kept between the tracks to ensure a good functioning of the sensor with polyaniline film since Vicentini, et al. [15] found that PAni conductive paths are not clear since PAni particles can form agglomerates resulting in isolated large conductive islands. The size of each sensor plate is set at $10 \mathrm{~mm}$ x $15 \mathrm{~mm}$. An initial copper layer of $35 \mathrm{~mm}$ thick was used.

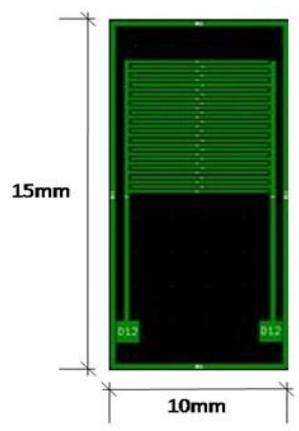

Figure 1: Layout of the designed PCB used as substrate of the gas sensors.

A layer of nickel and gold on the copper surface (ENIG-Electroless Nickel Immersion Gold treatment) was deposited (This activity was also in charge of the company Casa do Circuito) to prevent copper corrosion in the electrodes since the substrate would be put in contact with ammonium persulfate during the aniline polymerization.

In situ chemical synthesis of PAni was conducted following the procedure used by Dannetun et al. [16]. One solution composed by $0.574 \mathrm{~g}\left(\mathrm{NH}_{4}\right)_{2} \mathrm{~S}_{2} \mathrm{O}_{8}$ dissolved in $40 \mathrm{ml}$ of $1 \mathrm{M} \mathrm{HCl}$ solution at ice bath temperature $\left(0^{\circ} \mathrm{C}\right)$ was added dropwise to another solution composed by $1 \mathrm{ml}$ aniline dissolved in $60 \mathrm{ml}$ of 1 $\mathrm{M} \mathrm{HCl}$ solution and then stirred for a certain time, hereinafter referred to as the synthesis time of the conductive polymer film on the substrate of the gas sensor.

The chemical synthesis process of the HCl-doped PAni film on the substrate was reproduced three times for three synthesis times of 30, 60 and 90 minutes, respectively. For each synthesis time, a group of five PCBs was used, resulting in fifteen sensors. In all, ammonium persulfate was used as the oxidant agent. According to Ayad et al. [17] there is a direct relationship between synthesis time and the film thickness. After, the PCBs were washed with $1 \mathrm{M} \mathrm{HCl}$ solution, dried in a vacuum oven and then they were allocated in a desiccator with silica gel for 48 hours.

\subsection{PAni and ethanol sensor characterization}

Scanning electron microscopy (SEM) was performed to investigate the thickness of the copper and the conductive PAni film, both deposited on the PCB substrate, as well as to identify the morphology of the PAni film. The electrodes were metallized with gold plasma and were placed in JEOL JSM Model 65 / OLV 
electron microscope with EDS. The SEM analyses were performed at $10 \mathrm{kV}$.

Fourier transform infrared spectrophotometry (FTIR) were performed using the Nicolet spectrophotometer IS10 system with Smart OMW transmission spectroscopy and internal reflection or attenuated total reflectance (ATR) probe with diamond. This analysis was used to determine the chemical structure of PAni and its possible changes due to exposure to ethanol. Two samples were prepared for these analyses: a sample of PAni synthesized with $\mathrm{HCl}$ for one hour and another one of PAni synthesized with $\mathrm{HCl}$ for one hour, mixed and stirred with anhydrous ethanol for 40 minutes.

The sensors were exposed to ethanol at different concentrations and electrical resistance measurements were made on direct current. For these measurements, sealed glass containers have been employed with silica gel (as a desiccator) for the preservation of sensors. In the inner part of the recipient lid, five sensors have been glued (Figure 2). In each sensor was soldered a cable with RCA connector for measuring through two wires. A glass container was used for exposure to ethanol with a sealed lid and 400 $\mathrm{mL}$ of ethanol. At the time of exposure, the sensors are removed from the desiccator and placed in the container with ethanol in the region that forms a static headspace with gas emanating from ethanol. The sensors are packaged in the sealed chamber until saturation and the formation of the headspace, where the analyses come into equilibrium concentrations.

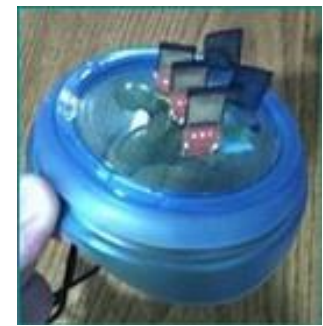

Figure 2: Sensors placed inside of the sealed lid of the desiccator.

A series of electrical resistance measurements of the PAni film were carried out in five fixed

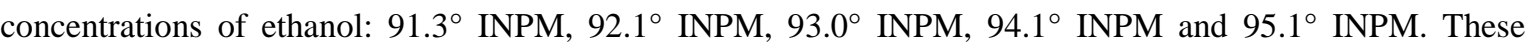
concentrations were adjusted in each vessel using a densimeter with temperature measurement and a density conversion table for INPM degrees as function of temperature. The electrical resistance measurements followed these procedures: first, simultaneous exposure of a group of five sensors to a determined ethanol concentration for two minutes and after they were exposure to clean and dry air within the desiccator for two more minutes. The electrical resistance of the samples was measured at room temperature using an $\mathrm{R}-\mathrm{C}$ meter Tenma, model 72-8150. From measured values of electric resistance, electrical conductivity $(\sigma)$ of the PAni films were calculated using the equation 1 , where $\mathrm{R}$ is the electrical resistance, $\mathrm{S}$ the cross-section area and $L$ the length of the sensor.

$$
\sigma=\frac{L}{(R \cdot S)}
$$

\section{RESULTS AND DISCUSSION}

This section presents all the obtained results with a discussion of their meaning, based on a statistical analysis and information from literature.

\subsection{Sensor characterization}

As substrate for the sensors, twenty PCBs were fabricated with interdigitated electrodes (Figure 3). 


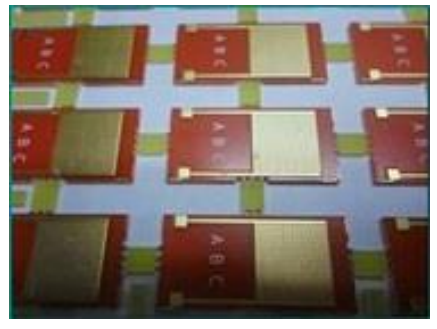

Figure 3: Glass fiber reinforced epoxy resin substrate with interdigitated electrodes of copper covered with nickel and gold (ENIG).

The initial thickness of copper was $35 \mu \mathrm{m}$ (prior to corrosion) on the PCB. After the corrosion of copper for the formation of interdigitated electrodes, a $4.33 \mu \mathrm{m}$-thick nickel layer was electroplated on copper and then a $0.07 \mu \mathrm{m}$-thick layer of gold was deposited over nickel with a theoretical average value of $39.4 \mu \mathrm{m}$ of total layer thickness. This activity was performed by the Casa do Circuito Company, using X-Ray Fluorescence with X - Ray Fischer equipment, model XDAL. A total layer thickness of $28.8 \mu \mathrm{m}$ was identified by SEM (Figure 4), i.e., copper was reduced to approximately $10.6 \mu \mathrm{m}(26.9 \%)$, supposedly, due to corrosion.

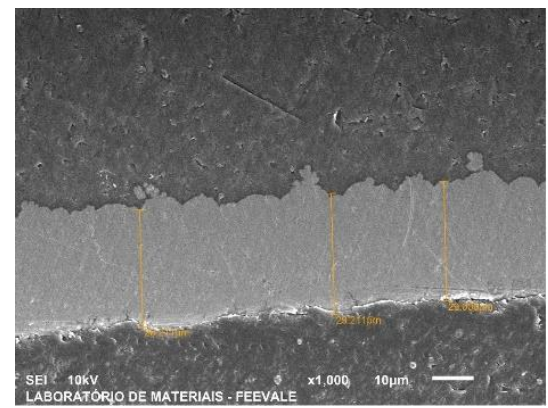

Figure 4: SEM photomicrograph of a cross-section of sensor substrate for thickness measurement of the total (copper + nickel + gold) layer.

Figure 5 illustrates the sensor obtained after the deposition of doped PAni film, that is, a protonic doped film and electrical conductor in the form of the green colored emeraldine salt where most of the imine nitrogens are protonated. Figure 6 shows the arrangement of the different layers of the sensor.

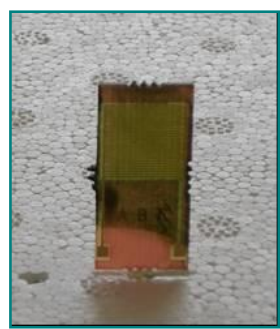

Figure 5: In situ deposition of PAni on sensor surface.

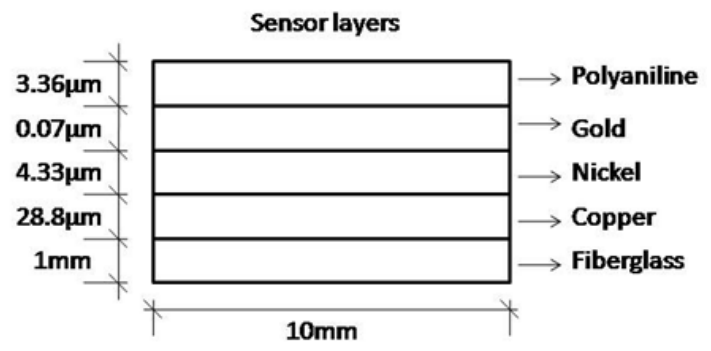

Figure 6: Sensor layers. 


\subsection{PAni Film Characterization}

An average thickness of 3.4 um of PAni film (polymerization time of 30 minutes) was identified by SEM analysis (Figure 7a). The morphological analysis of polyaniline by SEM indicated the presence of PAni nanofibers (Figure 7b). These fibers had diameters in the range 30-200 nm. Mikhaylov et al. found fiber diameters of the same order of magnitude [13]. Wang et al. [18] observed that the formation of nanofibers morphology in PAni could be obtained by oxidative chemical synthesis and reported the same nanofiber agglomerates through SEM analysis for PAni chemically synthesized with $1 \mathrm{M} \mathrm{HCl}$ and ammonium persulphate.

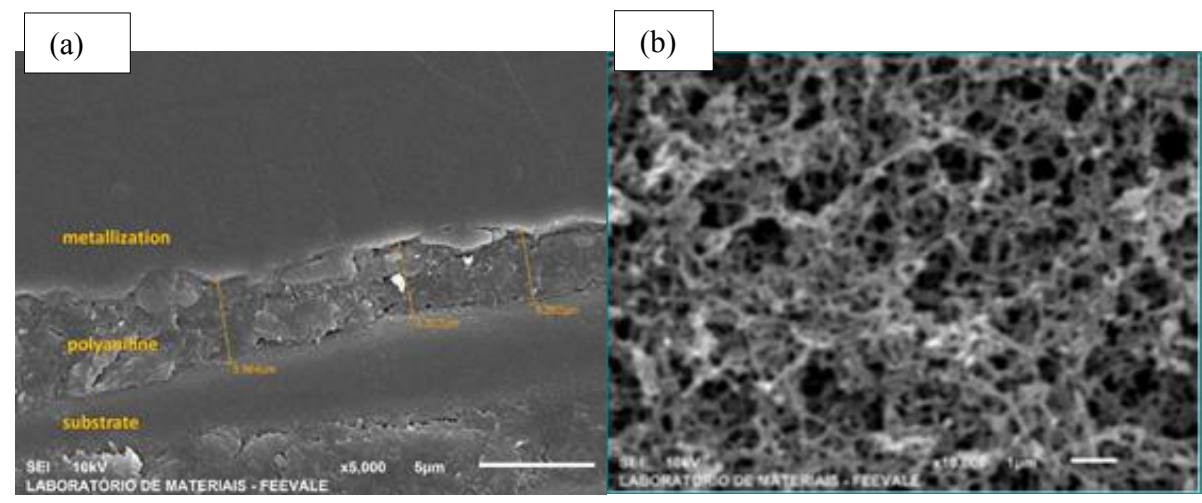

Figure 7: SEM photomicrographs of PAni films:(a) thickness of PAni film on the sensor and (b) surface morphology (for (a) an (b) deposition time 30 minutes in acid medium).

Regarding FTIR spectroscopy of PAni films, the same spectral bands were observed for both: the $\mathrm{HCl}$ doped PAni and for $\mathrm{HCl}$ doped PAni and mixed with anhydrous ethanol for 40 minutes (Figures 8 and 9, respectively), evidencing the band assigned to PAni in its conductive state (near $1150 \mathrm{~cm}-1$ ). Thus, no changes in chemical structure of PAni are noticeable by FTIR upon exposure to ethanol. This fact corroborates the slight decrease in electrical resistance of PAni sensors when exposed to anhydrous ethanol as further described. The characteristic bands of the conductive $\mathrm{HCl}$ doped PAni found were:

- The main bands at 1570 and $1490 \mathrm{~cm}^{-1}$ may be associated to $\mathrm{C}=\mathrm{C}$ bonds stretching in both quinoid and benzenoid rings. The bands at 1300 and $1240 \mathrm{~cm}^{-1}$ are attributed to $\mathrm{C}-\mathrm{N}$ bond stretching [19].

- The strong band around $1150 \mathrm{~cm}^{-1}$ is described as "electronic band" and is considered as a measure of electron delocalization degree and thus is a characteristic band of conductive polyaniline [20].

- The band around $810 \mathrm{~cm}^{-1}$ is assigned to the $-\mathrm{C}-\mathrm{H}$ out-of-plane bending vibrations in the aromatic ring [15].

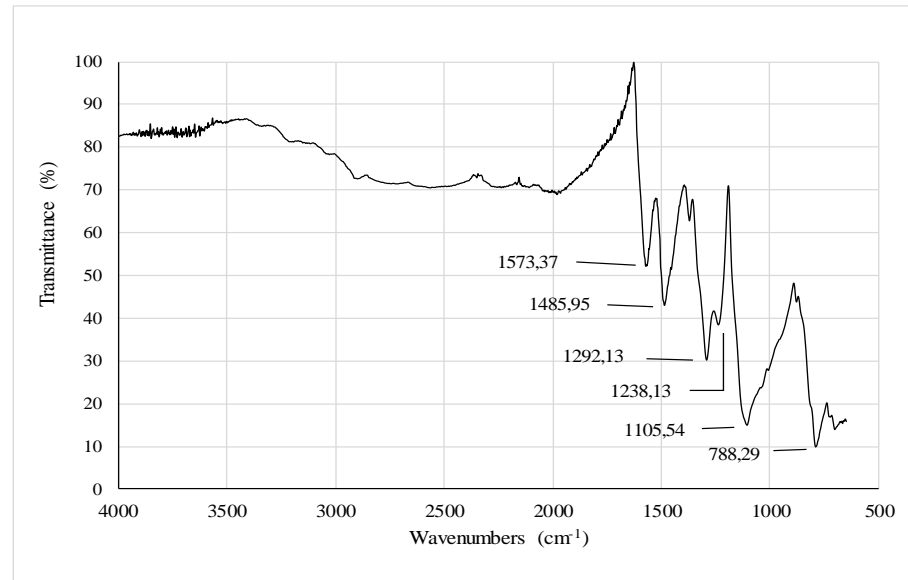




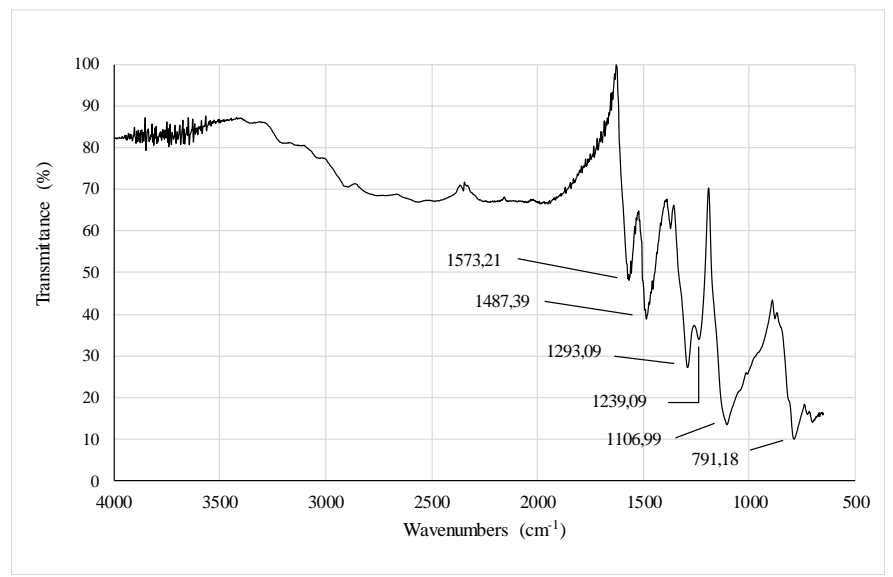

(b)

Figure 8: FTIR (ATR) spectrum of (a) $\mathrm{HCl}$ doped PAni (doped for an hour) and (b) HCl doped PAni (doped for one hour and mixed with anhydrous ethanol for 40 minutes).

The addition of volatile compounds to the sensor surface changes the electron flow in the system and therefore the sensor resistance. The volatiles may interact with at least (i) the polymer itself, (ii) the counterion or (iii) the solvent [11]. According to Tarachiwin et al. [21], when $\mathrm{HCl}$ doped PAni films are exposed to ethanol, their specific conductivity increases. The mechanism for this increased conductivity is due to the transfer of $\mathrm{H}^{+}$protons among the polyaniline chains. The same mechanism can be attributed to exposure to water vapor. This transfer of protons $\mathrm{H}^{+}$between the chains may result in: (a) protonation of quinoid rings of PAni which have not yet protonated and (b) an increase in mobility of charges between the chains of polyaniline.

\subsection{Measurements of electrical resistance of the sensors}

The conductivity of five sensors constructed from the synthesis of the conductive polymer film on PCBs (referred as sensor A, B, C, D and E) with 30 minutes of synthesis time, was determined. Table 1 shows the calculated values of the initial conductivity of the sensors constructed, right after the polymerization. The protonation of the PAni emeraldine base to the conductive form of PAni (emeraldine salt) promotes a conductivity increase from approximately $10^{-10} \mathrm{~S}_{\mathrm{cm}} \mathrm{cm}^{-1}$ to values greater than $1{\mathrm{~S} . \mathrm{cm}^{-1}}^{-1}$ a value comparable to that found in semiconductors [22]. According to Mello et al. [23], the electrical conductivity of PAni films,

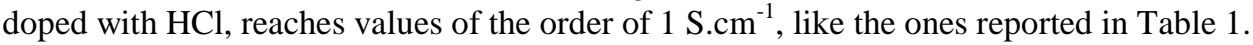

Table 1: Calculated values of initial conductivity of sensors A, B, C, D, E.

\begin{tabular}{c|c|c}
\hline Sensor & Electrical resistance $(\mathrm{k} \Omega)$ & Electrical conductivity $\left(\mathrm{S} . c m^{-1}\right)$ \\
\hline $\mathrm{A}$ & 2.7 & 1.0 \\
\hline $\mathrm{B}$ & 2.0 & 1.4 \\
\hline $\mathrm{C}$ & 2.4 & 1.2 \\
\hline $\mathrm{D}$ & 1.7 & 1.7 \\
\hline $\mathrm{E}$ & 2.2 & 1.3 \\
\hline
\end{tabular}

The electrical resistance reported in Table 1 was measured by exposing the sensors to the open air immediately after the deposition of the polyaniline film and storage in a desiccator for 24 hours.

Hong-Xing et al. [24] showed that the electrical resistance of PAni films under exposure to ethanol increases sharply at the first moment and falls quickly, demonstrating that this conductor polymer is more sensitive to ethanol than water. A similar experiment was performed using a sensor obtained by the in-situ deposition of PAni. The electrical resistance of the sensor was measured for about 15 minutes, immediately after a drop of ethanol was dripped onto the surface of the PAni film sensor through a data acquisition system which records the PAni electrical resistance each second (Figure 9). In figure 9, the measured electrical resistance 
values $(\mathrm{R})$ are referred to the initial resistance value (R0) by the R/R0 ratio. It is observed that the R/R0 ratio increases rapidly in the first minute. According to Hong-Xing et al. (2010), this is due to the rapid volume expansion of amorphous regions of PAni. In the next three minutes, the R/R0 ratio falls again due to faster evaporation of the ethanol molecules than of the water. The behavior of the electric resistance of the sensor is like that observed in the experiment by Hong-Xing et al. (2010), although the results here were obtained with an ohmmeter (two-point method) from the interdigitated electrode terminals covered by a PAni film [24].

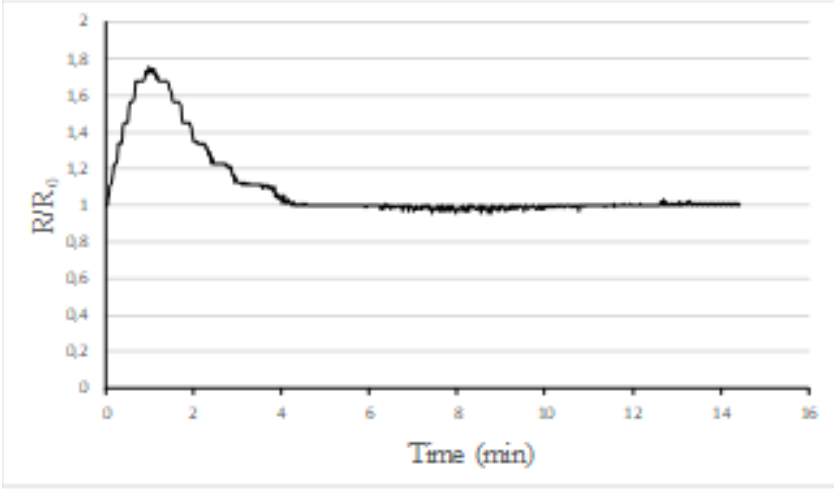

Figure 9: Electrical response of a PAni film sensor exposed to ethanol.

Further PAni film electrical resistance measurements for the group of five sensors synthesized for 30 minutes in acid medium were performed too. Each sensor was exposed to five different concentrations of ethanol: $91.3^{\circ}$ INPM, 92.1 ${ }^{\circ}$ INPM, 93.0 ${ }^{\circ}$ INPM, 94.1 ${ }^{\circ}$ INPM and 95.1 ${ }^{\circ}$ INPM.

The electrical resistance measurements were performed in two steps. First, the electrical resistance of the sensor was measured after exposure to dry air (inside the desiccator) for two minutes. Secondly, the sensor was exposed to ethanol solution with $91.3^{\circ}$ INPM concentration for two minutes and new electrical resistance measurements were recorded. These steps were repeated ninety times for the same ethanol concentration. After the first sequence of nine pairs of measurements (alternating dry air and $91.3^{\circ}$ INPM ethanol exposures), a new sequence of nine pairs of measurements were performed (now dry air exposure intercalating with $92.1^{\circ}$ INPM ethanol exposure) and so on up to $95.1^{\circ}$ INPM ethanol concentration. The same procedure was performed with the other four sensors.

It was observed that each of the sensors differentiated the ethanol concentrations, presenting different electrical resistance values which are directly proportional to the ethanol concentration. Considering the five sensors, the overall mean value of electrical resistance for each alcoholic concentration is $(65.6 \pm 1,6) \mathrm{kohms}$, $(73.2 \pm 1,9)$ kohms, $(94.3 \pm 2,5)$ kohms, $(109.4 \pm 2,7)$ kohms and $(133.4 \pm 3,5)$ kohms for the ethanol concentrations of $91.3{ }^{\circ} \mathrm{INPM}, 92.1^{\circ} \mathrm{INPM}, 93.0^{\circ} \mathrm{INPM}, 94.1^{\circ} \mathrm{INPM}$ and $95.1^{\circ} \mathrm{INPM}$, respectively.

In order to evaluate the variation in electrical resistance measurements of the sensors for different concentrations of ethanol, one-way ANOVA analysis were performed (Table 2) with significance level of 5\% for sensor A exposed to five different concentrations of ethanol. The value of the calculated $\mathrm{F}$ test (ratio between the variance between sample groups and the variance within the sample groups) is significant because it has a value greater than the table. Therefore, it is concluded that the variability between the groups is large, that is, there are large differences between the sample means of the groups that are not due solely to sample fluctuations. Then, the sensors are not suitable for reliable measurements of electrical resistance. The results presented in Table 2 showed that the sensors tested are not suitable for reliable measurements of electrical resistance since they cannot differentiate ethanol concentrations at a significance level of $5 \%$.

Table 2: One-way ANOVA analysis at a significance level of 5\% for sensor A as function of ethanol concentrations. 


\begin{tabular}{c|c|c|c|c|c|c}
\hline SOURCE & SQ & GDL & MQ & $\begin{array}{c}\text { TEST "F" } \\
\text { CALCULATED }\end{array}$ & $\begin{array}{c}\text { TEST "F" } \\
\text { TABLE5\% }\end{array}$ & RESULT \\
\hline Between Groups & 18253 & 4 & 4563 & 6,58 & 2,61 & Rejection H0 \\
\hline Inside Groups & 27731 & 40 & 693 & & & \\
\hline Total & 45985 & 44 & & & & \\
\hline
\end{tabular}

Legend: SQ - the sum of squares; GDL - the degrees of freedom between groups (first row) and the degrees of freedom within groups (second row). MQ - the square mean between groups in the first row and the square mean within the groups in the second row.

\section{CONCLUSION}

The sum of the thickness of the copper and the anticorrosive coating (ENIG) on the PCB measured by SEM agrees with the manufacturer's specifications. As the electrically conductive PAni films were not homogeneous in morphology since they were obtained by chemical synthesis, the smallest gap possible between the interdigitated electrodes of the substrate was used to ensure a larger contact area of the polyaniline film with the electrodes. The spacing of the interdigitated electrodes covered by electrically conductive PAni films act as variables resistors connected in series. The thickness of the conductive PAni film, obtained by in situ polymerization, is compatible with the thickness of PAni films reported in the literature.

FTIR analysis showed a band near $1140 \mathrm{~cm}^{-1}$ which is characteristic of the conductive polymer due to relocation caused by protonation. The data showed that the PAni films can be used in chemical sensors for measuring ethanol concentration. The presence of this band, both for $\mathrm{HCl}$ doped PAni and for $\mathrm{HCl}$ doped PAni mixed with anhydrous ethanol for 40 minutes, proves the fact reported in the literature that ethanol does not change the chemical structure of PAni.

The conductivity of the sensors was consistent with the work of other authors. When exposed to ethanol, a decrease in resistance of the sensors was found. The five tested sensors presented a response in terms of electric resistance which is directly proportional to the ethanol concentration. But the statistical evaluation of data (one-way ANOVA) identified a lack of selectivity of the sensor for different concentrations of ethanol in all measurements, i.e., the sensors have not been able to differentiate these ethanol concentrations. The sensor data show a similar function, indicating that there is potential for the application as ethanol sensors.

Many issues remain open and thus studies about the effect of different concentrations and types of oxidants and dopants of PAni on the sensor characteristics must be performed as well as the electrochemical deposition of PAni film and the analysis of the thickness influence of the PAni film on the selectivity of the sensor exposed to different gases.

\section{ACKNOWLEDGMENTS}

Authors are grateful to the Lutheran University of Brazil for financial support. We thank Profa. Prof. Cláudia Trindade Oliveira, from Feevale University, for orientation in MEV analysis and Damiane Paludo Budcke technique from the Polymer Laboratory of the University of Caxias do Sul, for her assistance in the FTIR tests.

\section{BIBLIOGRAPHY}

[1] BHADRA, S., CHATTOPADHYAY, S., SINGHA, N. K., KHASTGIR, D., "Improvement of conductivity of electrochemically synthesized polyaniline”, J. Appl. Polym. Sci., v.108, pp.57-64, 2008.

[2] PYO, M., HWANG, J.-H., "Conductivity changes of dodecylbezensulfonic acid-doped polyaniline during pressure loading/unloading", Synth. Met., v.159, pp.700-704, 2009.

[3] BAI, H., CHEN, Q., LI, C., et al., "Electrosynthesis of polypyrrole/sulfonated polyaniline composite films and their applications for ammonia gas sensing", Polymer, v. 48, pp. 4015-4020, 2007.

[4] KIM, J.-S., SOHN, S.-O., HUH, J.-S., "Fabrication and sensing behavior of PVF2 coated-polyaniline sensor for volatile organic compounds", Sensor Actuator B., v.108, pp. 409-413, 2005.

[5] GUIMARD, N. K., GOMEZ, N., SCHMIDT, C. E., "Conducting polymers in biomedical engineering", 
Prog. Polym. Sci., v. 32, pp. 876-921, 2007.

[6] BHADRA, S., KHASTGIR, D., SINGHA, N. K., et al., "Progress in preparation, processing and applications of polyaniline”, Prog. Polym. Sci., v. 34, pp. 783-810, 2009.

[7] MULlER, D., GARCIA, M., SALMORIA, G. V., et al., "SEBS/PPy.DBSA blends: Preparation and evaluation of electromechanical and dynamic mechanical properties", J. Appl. Polym. Sci., v.120, pp.351359, 2011.

[8] BOBER, P., STEJSKAL, J., TRCHOVÁ, M., et al., "Polyaniline-silver composites prepared by the oxidation of aniline with mixed oxidants, silver nitrate and ammonium peroxydisulfate: The control of silver content", Polymer, v. 52, pp. 5947- 5952, 2011.

[9] BHADRA, S., SINGHA, N. K., CHATTOPADHYAY, S., et al., "Effect of different reaction parameters on the conductivity and dielectric properties of polyaniline synthesized electrochemically and modeling of conductivity against reaction parameters through regression analysis", Journal of Polymer Science Part B: Polymer Physics, v. 45, n. 15, pp. 2046-2059, 2007.

[10] JAMES, D., SCOTT, S., ALI, Z. et al., "Chemical Sensors for Electronic Nose Systems", Microchim Acta., v. 149, pp. 1-17, 2005.

[11] SUJATHA, G., DHIVYA, N., AYYADURAI, K., et al., "Advances in Electronic - Nose Technologies", International Journal of Engineering Research and Applications (IJERA), v. 2, pp. 1541-1546, 2012.

[12] WU, H., CHEN, Z., ZHANG, J., et al., "Phthalocyanine-mediated non-covalent coupling of carbon nanotubes with polyaniline for ultrafast $\mathrm{NH}_{3}$ gas sensors", J. Mater. Chem. A 5, pp. 24493-24501, 2017.

[13] MIKHAYLOV, S., OGURTSOV, N., NOSKOV, Y., et al., "Ammonia/amines electronic gas sensors based on hybrid polyaniline-TiO2 nanocomposites. The effects of titania and the surface active doping acid", RSC Adv. 5 (2015) 20218-20226

[14] YOSHIOKA, N. A., ROCHA, H. L. R., CAZATI, T., et al., "Eletropolimerização pulsada para confecção de camadas transportadoras de cargas foto-geradas em dispositivos fotovoltaicos orgânicos", Polímeros Ciência e Tecnologia., v. 61, pp. 1-18, 2013.

[15] VICENTINI, D. S., BARRA, G. M. O., BERTOLINO, J. R., et al., "Polyaniline/thermoplastic polyurethane blends: Preparation and evaluation of electrical conductivity", European Polymer Journal., v. 43 pp. 4565-4572, 2007.

[16] DANNETUN, P., LAZZARONI, R., SALANECK, W.R., et al., "The electronic structure of emeraldine doped in situ from $\mathrm{HCl}$ in the gas phase as studied by photoelectron spectroscopy", Synthetic Metals., v. 41 pp. 645-648, 1991.

[17] AYAD, M. M., SALAHUDDIN, N., SHENESHIN, M. A., "Optimum reaction conditions for in-situ polyaniline films", Synthetic Metals., v.132, pp. 185-190, 2003.

[18] WANG, P.-C., VENANCIO, E.C., SARNO, D. M., et al., "Simplifying the reaction system for the preparation of polyaniline nanofibers: Re-examination of template-free oxidative chemical polymerization of aniline in conventional low-pH acidic aqueous media", Reactive \& Functional Polymers., v. 69, pp. 217-223, 2009.

[19] ABDOLAHI, A., HAMZAH, E., IBRAHIM, Z., et al., "Synthesis of Uniform Polyaniline Nanofibers through Interfacial Polymerization”, Materials (R.J.), v. 5, pp. 1487-1494, 2012.

[20] YILMAZ, F., KÜÇÜKYAVUZ, Z., “Solution properties of polyaniline”, Polymer International, v. 59 pp. 552-556, 2010.

[21] TARACHIWIN, L., KIATTIBUTR, P., RUANGCHUAY, L., et al., "Electrical conductivity of polyaniline films to ethanol-water mixtures", Synthetic Metals, v.129, pp. 303-308, 2002.

[22] MARIANO, L. C., SAlVATIERRA, R. V., CAVA, C.E., et al., "Electrical Properties of SelfAssembled Films of Polyaniline/Carbon Nanotubes Composites", The Journal of Physical Chemistry C., v. 118, pp. 24811-24818, 2014.

[23] MELLO, S.V., RIUL JR, A.., MATTOSO, L.H.C., et al., "Protonation Effects in polyaniline Langmuir films investigated by surface potential measurements", Synthetic Metals., v. 84, pp. 773-774, 1997.

[24] HONG-XING, Y., MENG-MENG, L., HE, Y., et al, "Electrical resistance response of polyaniline films to water, ethanol, and nitric acid solution”, Chinese Physics B - IOPscience. v. 19, pp.1-6, 2010. 